\title{
Icariin attenuates neuroinflammation and exerts dopamine neuroprotection via an Nrf2-dependent manner
}

\author{
Bei Zhang ${ }^{\dagger}$, Guoqing Wang ${ }^{\dagger}$, Jingyi He, Qiuyu Yang, Daidi Li, Jingjie Li and Feng Zhang ${ }^{*}$ (D)
}

\begin{abstract}
Background: Oxidative stress and neuroinflammation are considered the major central events in the process of Parkinson's disease (PD). Nrf2 is a key regulator of endogenous defense systems. New finds have contacted activation of Nrf2 signaling with anti-inflammatory activities. Therefore, the outstanding inhibition of neuroinflammation or potent Nrf2 signaling activation holds a promising strategy for PD treatment. Icariin (ICA), a natural compound derived from Herba Epimedii, presents a number of pharmacological properties, including anti-oxidation, anti-aging and anti-inflammatory actions. Recent studies have confirmed ICA exerted neuroprotection against neurodegenerative disorders. However, the underlying mechanisms were not fully elucidated.

Methods: In the present study, mouse nigral stereotaxic injection of 6-hydroxydopamine (6-OHDA)-induced PD model was performed to investigate ICA-conferred dopamine (DA) neuroprotection. In addition, adult Nrf2 knockout mice and primary rat midbrain neuron-glia co-culture was applied to elucidate whether ICA-exerted neuroprotection was through an Nrf2-dependent mechanism.

Results: Results indicated that ICA attenuated 6-OHDA-induced DA neurotoxicity and glial cells-mediated neuroinflammatory response. Furtherly, activation of Nrf2 signaling pathway in glial cells participated in ICA-produced neuroprotection, as revealed by the following observations. First, ICA enhanced Nrf2 signaling activation in 6-OHDAinduced mouse PD model. Second, ICA failed to generate DA neuroprotection and suppress glial cells-mediated proinflammatory factors production in Nrf2 knockout mice. Third, ICA exhibited neuroprotection in primary neuron-glia co-cultures but not in neuron-enriched cultures (without glial cells presence). Either, ICA-mediated neuroprotection was not discerned after Nrf2 siRNA treatment in neuron-glia co-cultures.
\end{abstract}

Conclusions: Our findings identify that ICA attenuated glial cells-mediated neuroinflammation and evoked DA neuroprotection via an Nrf2-dependent manner.

Keywords: Parkinson's disease, Neuroinflammation, Nrf2, Icariin, Neuroprotection

\section{Highlights}

- Oxidative stress and neuroinflammation are considered as the major central events in the process of Parkinson's disease

- Activation of Nrf2 signaling has been linked to antiinflammatory activities

\footnotetext{
* Correspondence: zhangf@zmc.edu.cn

${ }^{+}$Bei Zhang and Guoqing Wang contributed equally to this work.

Key Laboratory of Basic Pharmacology of Ministry of Education and Joint International Research Laboratory of Ethnomedicine of Ministry of Education, Zunyi Medical University, Zunyi, Guizhou, China
}

- Icariin attenuates glial cells-mediated neuroinflammation and evoked dopamine neuroprotection via an Nrf2-dependent manner

\section{Introduction}

Parkinson's disease (PD) is a multifactorial neurodegenerative disease accompanied by progressive loss of dopamine (DA) neurons in the midbrain substantia nigra (SN) [1]. The clinical manifestation of PD mainly includes dyskinesia, resting tremor, muscle rigidity, and gait disturbance [2]. Currently, there is no effective treatment for PD. 
Although the cause of PD is not completely clear, there is an age-related excessive oxidative stress, leading to neuroinflammation, DA auto-oxidation, a-synuclein accumulation, and glial cells activation [3, 4]. Nuclear factor erythroid-2 related factor 2 (Nrf2), a key regulator of redox homeostasis, controls acute/chronic inflammation [5, 6]. Nrf2 activation initiates phase II enzymes expressions, such as heme oxygenase-1 (HO-1) and NADPH quinone oxidoreductase 1 (NQO1), which mitigates the pathogenesis of neurodegenerative diseases [7]. However, insufficient Nrf2 activation has been highly involved in the progress of neurodegenerative diseases. In addition, the anti-inflammatory properties of Nrf2 are well established. Amounts of evidence presented a transcriptional repression of pro-inflammatory cytokines, including tumor necrosis factor- $\alpha$ (TNF- $\alpha$ ), interleukin- $1 \beta$ (IL-1 $\beta$ ), and nitric oxide (NO), in microglia and astroglia following Nrf2 activation $[1,8]$. Moreover, a Nrf2 activator, dimethyl fumarate (DMF)-mediated DA neuroprotection has been involved in inhibiting activation of microglia and astroglia [9]. Also, Nrf2-mediated neuroinflammation has been considered to the primary therapeutic targets for amyotrophic lateral sclerosis (ALS) $[10,11]$. Together, the outstanding inhibition of neuroinflammation or potent Nrf2 signaling activation holds a promising strategy for neurodegenerative diseases treatment.

Icariin (ICA) is a flavone compound extracted from Herba Epimedii and shows a range of pharmacological effects, such as anti-oxidant, anti-inflammation, and anti-aging actions $[12,13]$. Furtherly, ICA could pass the blood-brain barrier and alleviates inflammatory infiltration in rat models of neurological disorders. For example, ICA attenuated lipopolysaccharide (LPS)-induced learning and memory deficits in rats by inhibiting the production of pro-inflammatory factors in hippocampus [14]. In the PD animal model, ICA protected DA neuronal damage against LPS-induced neurotoxicity [15]. However, the specific target and the underlying mechanisms underlying ICA-elicited neuroprotection are not fully illuminated.

In the present study, we detected the neuroprotective effects of ICA against 6-hydroxydopamine (6-OHDA)-induced DA neurotoxicity both in vivo and in vitro. Furthermore, adult Nrf2 knockout mice and primary rat midbrain neuron-glia co-culture was validated to elucidate whether ICA-exerted DA neuroprotection was via an Nrf2-dependent mechanism.

\section{Materials and methods Reagents}

ICA (purity > 98\%) was purchased from Nanjing Zelang Biological Technology Co., Ltd. (Nanjing, China). 6-OHDA was obtained from Sigma-Aldrich (St. Louis, MO). Sytox green nucleic acid fluorescence stain was bought from Bio-Rad (Hercules, CA, USA). Anti-tyrosine hydroxylase $(\mathrm{TH}), \mathrm{HO}-1, \mathrm{NQO} 1$, glial cell line-derived neurotrophic factor (GDNF) and brain-derived neurotrophic factor (BDNF) antibodies were obtained from Abcam (Cambridge, MA, USA). Anti-Kelch-like ECH-associated protein 1 (Keap1), Nrf2, proliferating cell nuclear antigen (PCNA), ionized calcium-binding adapter molecule-1 (Iba-1), glia fibrillary acidic protein (GFAP), inducible nitric oxide synthase (iNOS), TNF- $\alpha$, and $\beta$-actin antibodies were bought from Proteintech Group (Chicago, IL, USA). Nrf2-siRNA and control-siRNA were purchased from Thermo Fisher Scientific (Waltham, MA, USA).

\section{Animals and treatment}

Male wild type (WT) mice and male homozygous Nrf2 knockout (Nrf2 KO) mice (25-30 g, 8-10 weeks) were purchased from the Model Animal Research Centre of Nanjing University (Nanjing, China). All experimental procedures were carried out in accordance with Chinese Guidelines of Animal Care and Welfare and this study received an approval from the Animal Care and Use Committee of Zunyi Medical University (Zunyi, China). WT and Nrf2 KO mice received intragastric administration with ICA $(60 \mathrm{mg} / \mathrm{kg})$ once daily for 10 consecutive days. On the third day, mice were injected stereotactically with 6 -OHDA ( $4 \mu \mathrm{g}$, in $0.2 \%$ ascorbic acid) in the $\mathrm{SN}$ on the left side of the brain with coordinates from Bregma: AP $-2.2 \mathrm{~mm}$, ML $1.4 \mathrm{~mm}, \mathrm{DV}-4.7 \mathrm{~mm}$ [16]. Normal control animals accepted equal volume saline.

\section{Rotarod test}

The rotarod test was widely used to detect the muscular coordination and balance [17]. Prior the test start, all mice were trained to stay at $0 \mathrm{rpm}$ for a while, then steadily increased to $10 \mathrm{rpm}$ in $30 \mathrm{~s}$ and $5 \mathrm{rpm}$ per $30 \mathrm{~s}$ until the mice slid off the steps. Animal behavioral activity was detected for 3 repeated trials on 1 day, and the average duration of stay on the rod was recorded.

\section{Open field test}

Open field test was performed at the last ICA application for evaluating the levels of anxiety emotionality of animals [18]. Mice were placed on the open field and each mouse was placed in a separate area and its behavioral parameters were recorded during the $5 \mathrm{~min}$. The device was washed with $75 \%$ alcohol solution before each next behavioral test in order to eliminate the odors from the previous mouse. The distance of mice in central and peripheral activities was recorded by computer. After the experiment, the total distance of mouse movement was counted. 


\section{Ultra-high-performance-liquid chromatography (UHPLC) analysis}

Mouse striatum levels of DA, DOPAC, and HVA were detected by Agilent 1290 Infinity II series UHPLC System (Agilent Technologies). Striatum tissues were sonicated in extract solvent (acetonitrile-methanol-water, 2:2:1, 2\% formic acid). The homogenate was centrifuged, and a $100 \mu \mathrm{l}$ aliquot of the clear supernatant was transferred to an auto-sampler vial for UHPLC-MS/MS analysis. The mobile phase A contained $10 \mathrm{mmol} / \mathrm{L}$ ammonium acetate/ $0.1 \%$ formic acid, and the mobile phase $\mathrm{B}$ was acetonitrile.

\section{Immunofluorescence staining}

Thirty-five-micrometer-thick brain sections stained with the corresponding antibodies. Briefly, brain slices were incubated with $0.3 \%$ Triton X-100 and closed with goat serum. Subsequently, brain slices concentrated with anti-TH (1:500), Iba-1 (1:200), and GFAP (1:300) antibodies at $4{ }^{\circ} \mathrm{C}$ overnight, respectively. Then, the slices were incubated with goat anti-rabbit antibody (green) secondary antibodies (1:1500) for $1 \mathrm{~h}$. Digital images of TH-positive neurons, Iba-1-positive microglia, and GFAP-positive astroglia were obtained via Olympus microscope (Olympus, Tokyo, Japan). DA damage was assessed through the quantification of $\mathrm{TH}$-positive neurons.

\section{Western blotting}

Nuclear and cytosol fractions were extracted from the mice midbrain tissues with Nuclear-Cytosol Extraction Kit (Solarbio, Beijing, China) following the manufacture's protocols. Total proteins were isolated by RIPA lysis solution (Solarbio, Beijing, China). Protein levels were quantified using BCA assay. Equal amounts of protein $(10-30 \mu \mathrm{g} /$ lane $)$ were separated on $10 \%$ Bis-Tris $\mathrm{Nu}$-PAGE gel. Then, the membranes were incubated with primary antibodies: TH (1:2000), Iba-1 (1:800), GFAP (1:1000), Nrf2 (1:1500), Keap1 (1:2000), HO-1 (1:10000), NQO1 (1:2000), PCNA (1:2000), TNF- $\alpha$ (1:800), iNOS (1:1500), GDNF (1:800), BDNF (1:1000), and $\beta$-actin (1:4000). Next day, the membranes were incubated with anti-rabbit IgG secondary antibodies at 1:5000 for $1 \mathrm{~h}$. Finally, the blots were detected using ECL substrate. $\beta$-actin was used as an internal standard to monitor loading errors. Densitometric analysis of immunoblots was performed using the Quantity One (Bio-Rad, Hercules, CA, USA) software system. The ratio of densitometry values of purpose protein with $\beta$-actin was analyzed and normalized to each respective control groups.

\section{Real-time RT-PCR assay}

The total RNA of mouse midbrain tissues was prepared using RNeasy kit and the detailed steps of Real-time RT-PCR were described previously [19]. Nrf2, Keap1,
HO-1, NQO1, and $\beta$-actin genes were tested. Accordingly, the genes' expression was normalized with $\beta$-actin. The primer sequences were as follows.

\begin{tabular}{|c|c|c|c|}
\hline Gene & Source & Forward primer $\left(5^{\prime}-3^{\prime}\right)$ & Reverse primer $\left(5^{\prime}-3^{\prime}\right)$ \\
\hline Nrf2 & Mouse & $\begin{array}{l}\text { CAGTCTTCACCAC } \\
\text { CCCTGAT }\end{array}$ & $\begin{array}{l}\text { CAGTGAGGGGATCG } \\
\text { ATGAGT }\end{array}$ \\
\hline Keap1 & Mouse & $\begin{array}{l}\text { AGGAATGAGTGGCG } \\
\text { GATGAT }\end{array}$ & $\begin{array}{l}\text { GCGCTCCACACTGT } \\
\text { TCAACT }\end{array}$ \\
\hline $\mathrm{HO}-1$ & Mouse & $\begin{array}{l}\text { AGAGGCTAAGACCG } \\
\text { CCTTCC }\end{array}$ & $\begin{array}{l}\text { TCTGACGAAGTGAC } \\
\text { GCCATC }\end{array}$ \\
\hline NQO1 & Mouse & $\begin{array}{l}\text { AGCCAATCAGCGTT } \\
\text { CGGTAT }\end{array}$ & $\begin{array}{l}\text { AGCCAATCAGCGTT } \\
\text { CGGTAT }\end{array}$ \\
\hline$\beta$-actin & Mouse & $\begin{array}{l}\text { GTGCTATGTTGCTCT } \\
\text { AGACTTCG }\end{array}$ & $\begin{array}{l}\text { ATGCCACAGGATTC } \\
\text { CATACC }\end{array}$ \\
\hline
\end{tabular}

\section{Primary rat midbrain neuron-glia and neuron-enriched} cultures

Female pregnant SD rats (250-300 g) were purchased from the Experimental Animal Center of the Third Military Medical University (Chongqing, China). Primary midbrain neuron-glia cultures were prepared from the ventral mesencephalic tissues of embryonic rats 14-15 days age [20]. Then, the rat whole brains were dissected and the midbrain were isolated. After excision of blood vessels and meninges, the midbrain tissues were mechanically triturated and the dissociated cells were planted in poly-D-lysine-coated 24-well plates or 96-well plates. Seven-day-old cultures were employed for drug treatment. During treatment, immunocytochemical staining demonstrated that primary neuron-glia co-cultures composed of $50 \%$ astrocytes, $10 \%$ microglia, $40 \%$ neurons, and $1 \%$ DA neurons. In addition, midbrain neuron-enriched cultures were prepared by adding cytosine $\beta$-D-arabinofuranoside $(8 \mu \mathrm{M})$ in primary neuron-glia cultures to inhibit glial cells proliferation [21]. ICA treatment was performed with 7 days of cultures followed by 6 -OHDA $(40 \mu \mathrm{M})$ intervention [15].

\section{Primary rat mixed-glia cell cultures}

Primary rat mixed-glia cultures were prepared from the whole brains of 1-day-old rat pups [22]. The meninges and blood vessels were isolated, the brain tissues were homogenized and the cells $\left(2 \times 10^{6} /\right.$ well $)$ were planted in a polyD-lysine-coated 6-well plate. Seven-day-old mixed-glial cells were cultured for drug treatment.

\section{Nrf2-siRNA transfection}

Primary mixed-glia cells were transfected with Nrf2-siRNA $(40 \mathrm{nmol} / \mathrm{L})$ or control-siRNA $(40 \mathrm{nmol} / \mathrm{L})$ for $24 \mathrm{~h}$. Western blotting and real-time RT-PCR were used to test the transfection rate. 
Neuron-glia reconstituted cultures using transwell

First, primary neuron-enriched cultures were planted in 24-well culture plates. Mixed-glia cultures were planted in the transwell. Seven days later, the mixed glial cells in transwell were processed by Nrf2-siRNA for 1 day. Then, the mixed glial cells were transferred to 7-day neuron-enriched cultures within fresh medium. Accordingly, the reconstituted neuron-glia cultures were treated with ICA and 6-OHDA for 7 days. DA neurons damage was assessed by DA neuronal quantification and $\mathrm{TH}$ protein expression detection.

\section{Immunocytochemical staining}

Cells were fixed with $4 \%$ paraformaldehyde followed by permeabilization using Triton X-100 and closed with goat serum. DA neurons were labeled with anti-TH (1:500) antibody at $4{ }^{\circ} \mathrm{C}$ overnight and then incubated with anti-rabbit-IgG (1:1500) antibody for $1 \mathrm{~h}$. TH-positive neurons numbers were calculated from three wells, and three randomly selected areas were analyzed for each group.

\section{Statistical analysis}

All data were presented as mean \pm SEM. Statistical comparisons were analyzed using GraphPad Prism 5 (GraphPad Software Inc., La Jolla, CA, USA) by one-way ANOVA. After ANOVA expressed significant differences, the Bonferroni's post hoc test was used for all pairwise comparisons among means. Statistical significance was considered as $p<0.05$.

\section{Results}

ICA ameliorated 6-OHDA-induced DA neuronal damage Neuroprotective effects of ICA on 6-OHDA-induced DA neuronal damage were investigated in WT mice. We first examined animal behavior changes via the rotarod and open-field tests. As shown in Fig. 1a and b, 6-OHDA reduced the time mice stayed on the rod and locomotor distance compared with control group. However, ICA attenuated 6-OHDA-induced decrease on these 2 tests. Furthermore, in both control and ICA alone group, strong $\mathrm{TH}$ immunoreactivity in midbrain $\mathrm{SN}$ was detected, whereas the $\mathrm{TH}$ immunoreactivity was interrupted in 6-OHDA group. ICA pretreatment protected DA neurons from 6-OHDA-induced neurotoxicity as shown by the enhanced TH-positive neurons number (Fig. 1c). The further quantification of DA neurons confirmed similar observations (Fig. 1d). As illustrated in Fig. 1e, compared with control group, 6-OHDA reduced striatum DA and DOPAC levels and had no significant effects on HVA content. Compared with 6-OHDA group, ICA increased DA concentration and did not attenuate the levels of HVA and DOPAC. Moreover, the DA metabolite ratio was increased in the 6-OHDA group and ICA treatment turned over this ratio (Additional file 1).

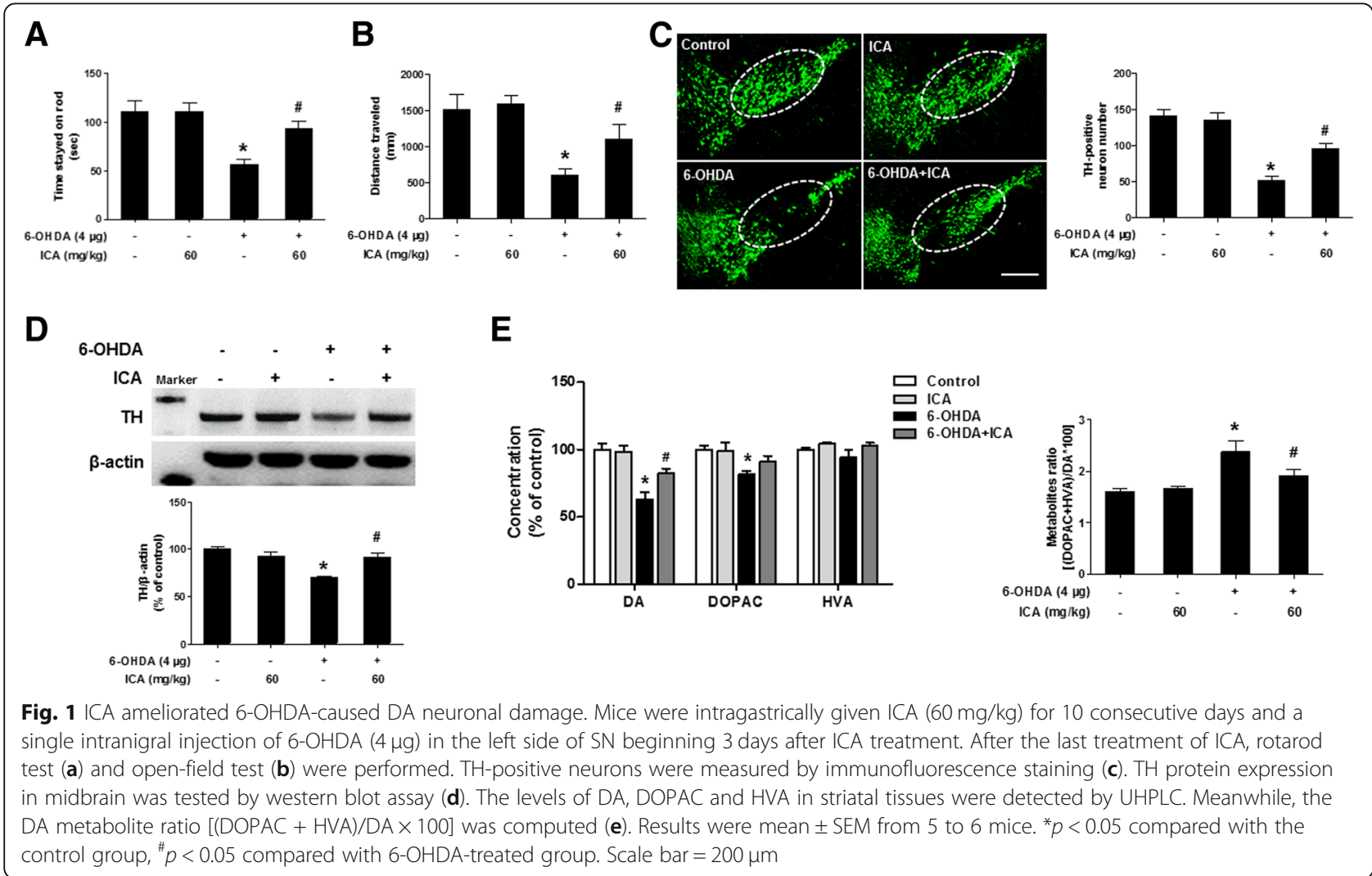




\section{ICA activated Nrf2 signaling pathway}

To confirm whether ICA could activate Nrf2 signaling pathway in mice midbrain $\mathrm{SN}$, the expression of Nrf2, Keap1, HO-1, and NQO1 were detected by RT-PCR and western blot assay. As shown in Fig. 2a, Nrf2 mRNA expression was upregulated in 6-OHDA and 6-OHDA+ICA treatment group. Meanwhile, Keap1, HO1, and NQO1 mRNA levels were more prominent after 6-OHDA alone or co-treatment with ICA exposure (Fig. 2b). Additionally, ICA increased the total protein expression of Nrf2. Furthermore, to further determine whether ICA could affect Nrf2 distribution, the cytosolic components and nuclear fractions were measured. We used the cytosolic marker HSP90 to verify the purity of nuclear proteins, the nuclear fraction was not contaminated by cytosolic proteins as confirmed by the absence HSP90. Next, western blot analysis showed that 6-OHDA and 6-OHDA combined with ICA increased the translocation of Nrf2 from the cytosol to the nuclear (Fig. 2c). Also, the higher protein expressions of Keap1, HO-1, and NQO1 were indicated in 6-OHDA and 6-OHDA+ICA groups than those in control and ICA alone groups (Fig. 2d).

\section{Nrf2 signaling participated in ICA-mediated neuroprotection}

Since ICA promoted Nrf2 distribution, Nrf2 KO mice were further conducted to evaluate the role of Nrf2 on ICA-mediated neuroprotection. Firstly, the Nrf2 knockout efficiency was tested by western blotting (Fig. 3a). Next, the gene and protein expressions of Keap1, HO-1, and NQO1 in midbrain were not altered in Nrf2 KO mice (Fig. 3b and c). Furthermore, ICA-ameliorated 6-OHDA-induced decrease of time stayed on stick and locomotor distance was not discerned in Nrf2 $\mathrm{KO}$ mice (Fig. 3d and e). Consistently, ICA-mediated DA neuroprotection was absent in Nrf2 $\mathrm{KO}$ mice via $\mathrm{TH}$ immunostaining and protein level detection (Fig. 3f and g).

Interestingly, as shown in Fig. 1c, 6-OHDA injection caused $62 \%$ DA neuron loss $(54.0 \pm 4.8 \mathrm{TH}$-positive neurons) in WT mice. While in Fig. 3f, 6-OHDA injection enhanced the neuronal loss into $71 \% \quad(38.6 \pm 3.4$ $\mathrm{TH}$-positive neurons) in Nrf2 $\mathrm{KO}$ mice. In addition, attenuation of DA metabolite level and ratio produced by ICA in Nrf2 KO mice appeared to be less evident than those in WT mice (Fig. 3h).

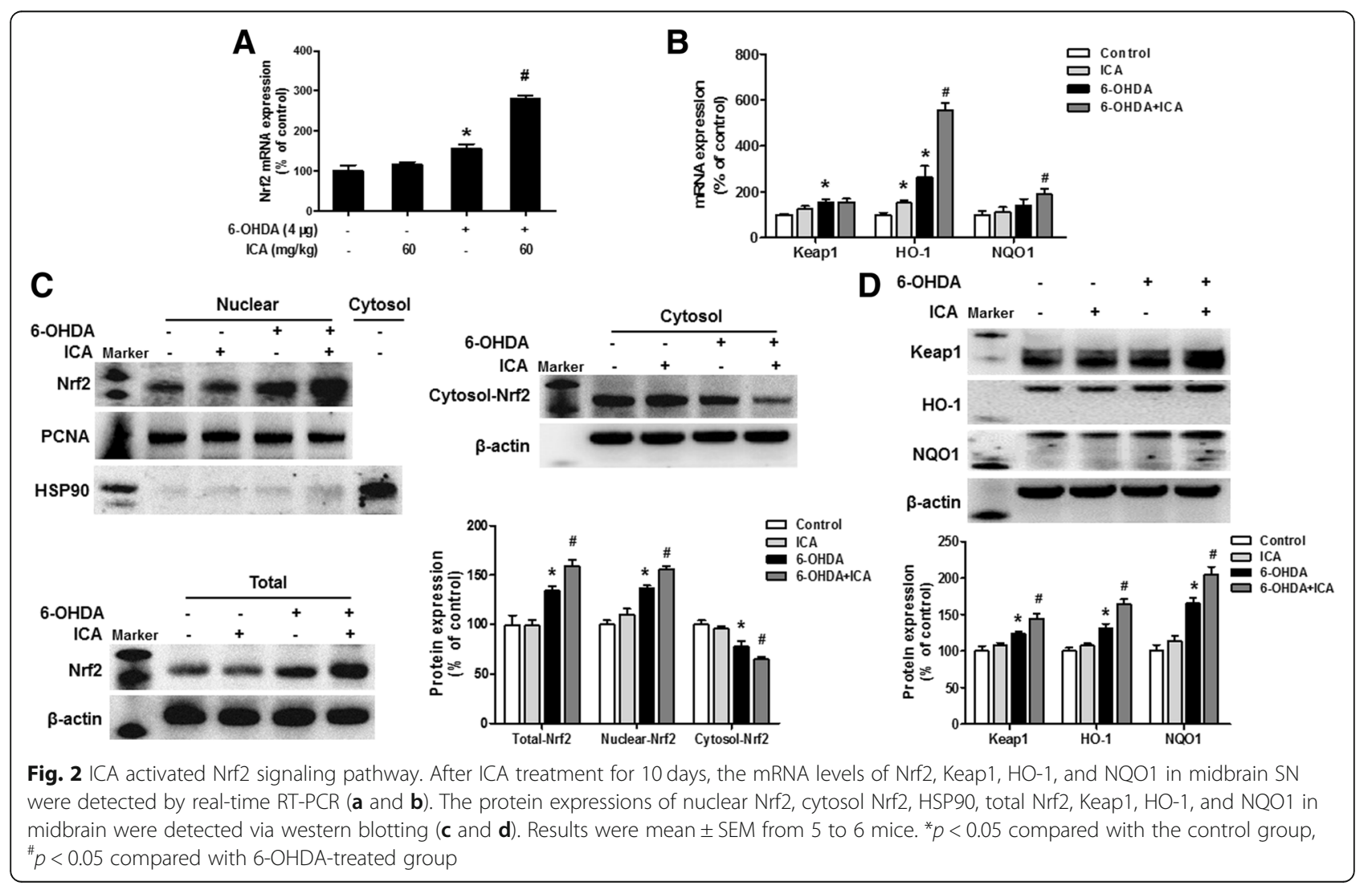




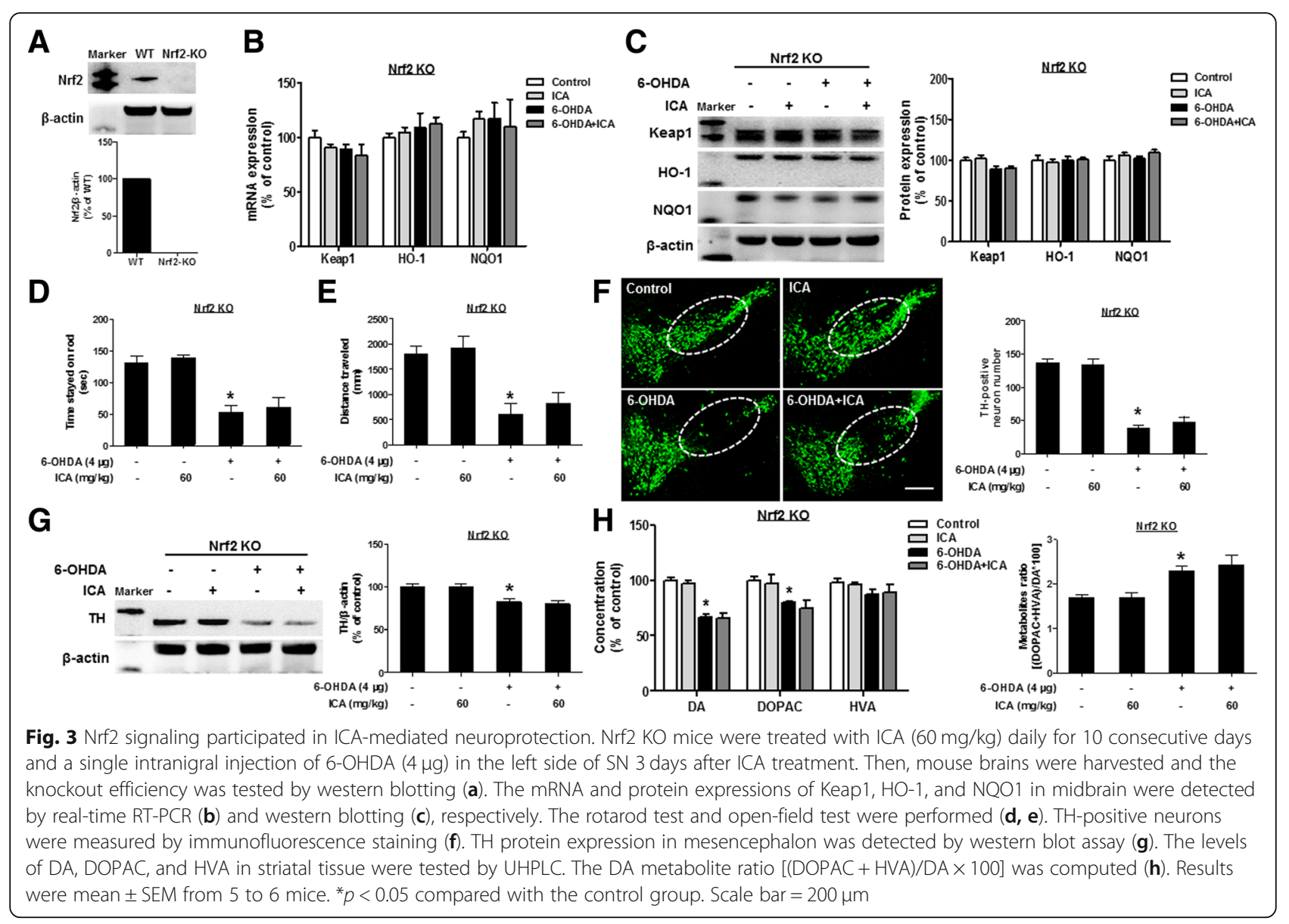

\section{ICA depressed glia cells-induced neuroinflammation through inhibiting Nrf2 signing activation}

It has been confirmed that glial cells-induced neuroinflammation participated in the process of DA neurodegeneration. Then, we detected the effects of ICA on neuroinflammation in 6-OHDA-caused DA neuronal damage. As indicated in Fig. 4a and c, ICA attenuated 6-OHDA-induced microglia and astroglia activation as evidenced by the less immunoreactivity of Iba-1 and GFAP shown in WT mice, whereas no obvious effects of ICA on the immunoreactivity in glial cells after 6-OHDA administration were discerned in $\mathrm{Nrf} 2 \mathrm{KO}$ mice. Meanwhile, Iba-1 and GFAP protein expression changes were parallel with the immunocytochemical analysis in WT and Nrf2 KO mice (Fig. 4b and d). Next, the actions of ICA on the function of glial cells were evaluated. As shown in Fig. 5a, ICA-induced initial decrease in TNF- $\alpha$ and iNOS protein expressions was observed compared with 6-OHDA group in WT mice. However, ICA failed to reduce TNF- $\alpha$ and iNOS protein levels after 6-OHDA stimulation in Nrf2 $\mathrm{KO}$ mice. On the other hand, both ICA and/or 6-OHDA had no significant effects on GDNF and BDNF production in WT mice and Nrf2 KO mice (Fig. 5b).

\section{ICA targeted glia cells Nrf2 to produce neuroprotection actions}

To further explore which cell type ICA targeted to produce DA neuroprotection via the activation of Nrf2 signaling, primary rat midbrain neuron-glia cultures and neuron-enriched cultures were applied, treated with ICA for $30 \mathrm{~min}$, and then stimulated by 6-OHDA. Seven days later, $\mathrm{TH}$ protein expression detection showed that ICA substantially attenuated 6-OHDA-induced DA neuronal injury in neuron-glia co-cultures, whereas no neuroprotection mediated by ICA was exhibited in neuron-enriched cultures, suggesting that glial cells were responsible for ICA-conferred DA neuroprotection (Fig. 6a). Subsequently, we examined whether ICA-mediated neuroprotection was attributable to the regulation of glial cells Nrf2 signaling. First, primary mixed-glial cells planted in transwell were processed with Nrf2-siRNA for $24 \mathrm{~h}$. When Nrf2-siRNA was processed, the Nrf2 gene and protein levels were downregulated and the silence rate was approximately 75.5\% (Fig. 6b). Then, Mixed-glial cells after silence were transferred to neuron-enriched cultures. Thus, the reconstituted neuron-glia cultures were established and treated with ICA and/or 6-OHDA for 7 days. DA neurons immunostaining and $\mathrm{TH}$ protein expression measurement 


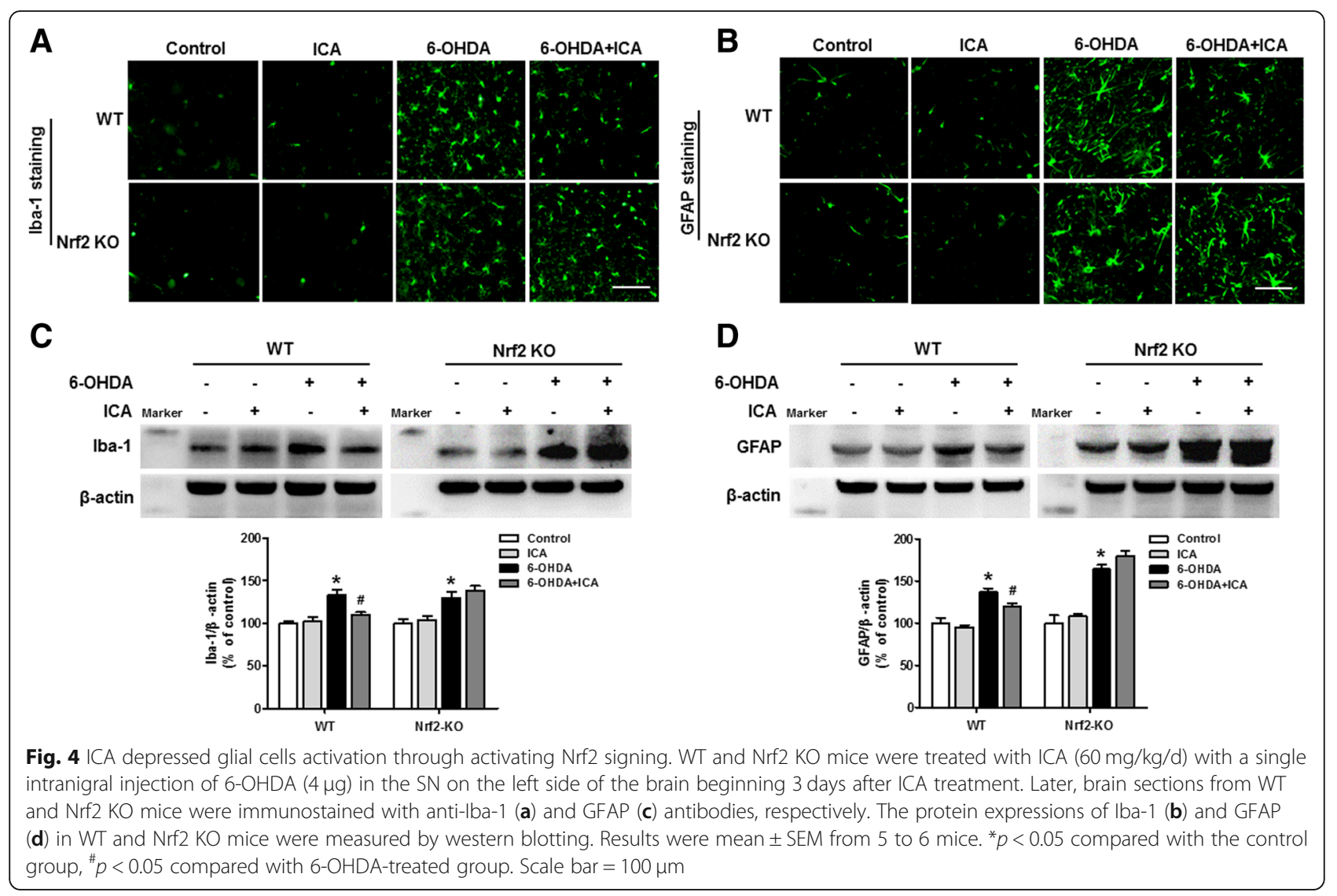

indicated that there was no significant difference among the control, Nrf2-siRNA, control-siRNA, and ICA alone cultures. In addition, 6-OHDA-induced reduction in DA neuronal number and $\mathrm{TH}$ protein expression was ameliorated by ICA pretreatment. However, the addition of Nrf2-siRNA to glial cells neutralized ICA-protected DA neurons against 6-OHDA-attended neurotoxicity (Fig. 6c and d). Collectively, ICA activated Nrf2 signaling in glial cells to exert DA neuroprotection.

\section{Discussion}

This study aimed at investigating the neuroprotective effects of ICA on 6-OHDA-induced DA neurotoxicity in vivo and the underlying mechanisms. Results demonstrated that ICA attenuated 6-OHDA-induced DA neurotoxicity and glia cells-mediated neuroinflammatory response. Furtherly, activation of Nrf2 signaling pathway in glial cells participated in ICA-produced neuroprotection, as revealed by the following observations. First, ICA enhanced Nrf2 signaling activation in 6-OHDA-induced mouse PD model. Second, ICA failed to generate DA neuroprotection and suppress glia cells-mediated pro-inflammatory factors production in Nrf2 knockout mice. Third, ICA exhibited neuroprotection in primary neuron-glia co-cultures but not in neuron-enriched cultures. Also, ICA-mediated DA neuroprotection was not discerned after Nrf2 siRNA treatment in neuron-glia co-cultures. Together, ICA inhibited glia cells-mediated neuroinflammation and afforded DA neuroprotection via the activation of Nrf2 signaling.

Maintainment of redox homeostasis in the CNS is dispensable for neuronal survival and development. Oxidative stress is generated by a continuous imbalance between the production and removal of reactive oxygen species (ROS) and reactive nitrogen species (RNS) [23]. The brain is recognized to be particularly sensitive to oxidative stress due to its high oxygen consumption and lipid level. In addition, oxidative stress serves as one of the major events participated in neuronal injury and loss in various chronic neurodegenerative diseases, such as Alzheimer's disease (AD), Parkinson's disease (PD), and Huntington's disease (HD) [24]. The transcription factor Nrf2 is considered to be a master mediator of cellular homeostasis, since it controls the expressions of multiple cytoprotective genes [25]. The list of these cytoprotective genes is persistently growing to include those participated in biotransformation, metabolic reprogramming, and anti-oxidant defense. However, Nrf2 deficiency replicated transcriptomic changes in AD patients [26]. Also, Nrf2 inactivation associated with aging was the main risk factor for PD [27]. Moreover, the protective effects of Nrf2 signaling activation on ischemic injury was not indicated in Nrf2 knockout mice either [28]. 


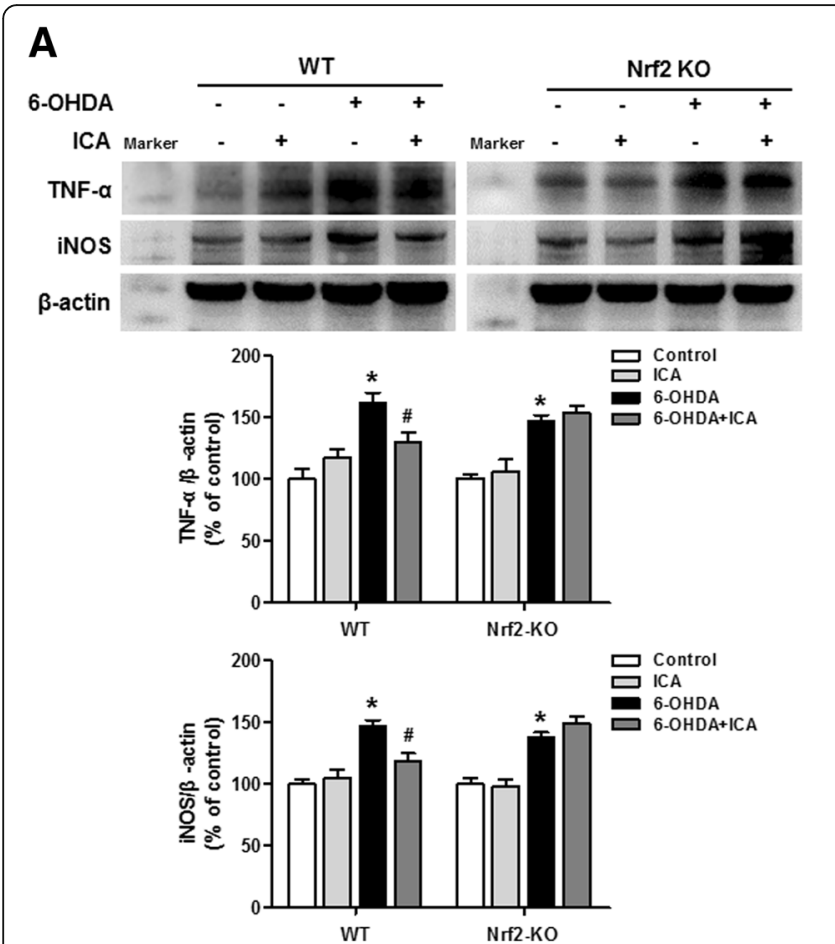

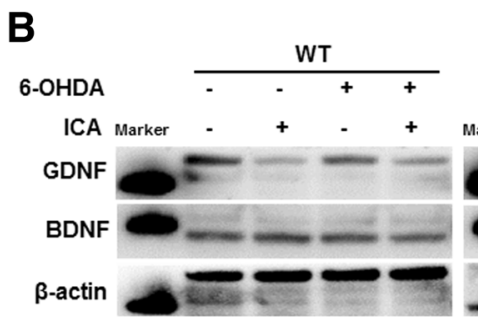
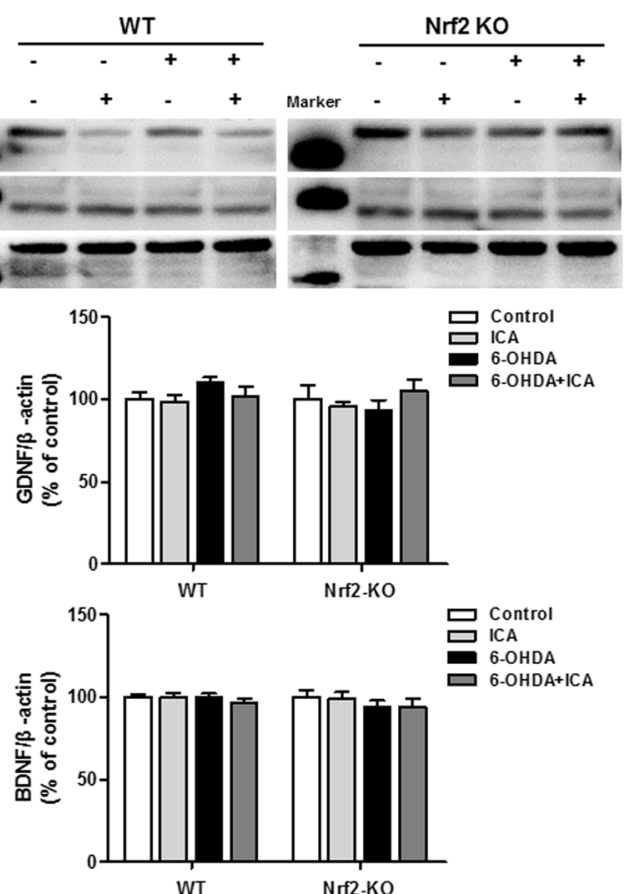

WT

Fig. 5 The role of Nrf2 signaling on ICA-affected functions of glial cells. After ICA treatment for 10 days, the protein levels of pro-inflammatory mediators, such as TNF-a and iNOS (a), and the production of neurotrophic factors, such as GDNF and BDNF (b), in the midbrain of WT and Nrf2 $\mathrm{KO}$ mice were measured by western blotting. Results were mean \pm SEM from 5 to 6 mice. ${ }^{*} p<0.05$ compared with the control group, ${ }^{*} p<0.05$ compared with 6-OHDA-treated group

Therefore, a functional Nrf2 signaling is identified as an important neuroprotective regulator of oxidative stress-related neurodegenerative disorders. In this study, ICA protected DA neurons against 6-OHDA-induced neurotoxicity, accompanied by the activation of Nrf2 signaling pathway. Furthermore, ICA failed to exert DA neuroprotection in Nrf2 knockout mice and primary rat midbrain neuron-glia co-culture treated by Nrf2 siRNA. Together, these results strongly suggested ICA-mediated DA neuroprotection might be attributable to the activation of Nrf2 signaling.

It is interesting to note that ICA targeted glia cells to exhibit neuroprotection as evidenced by this protection shown in primary neuron-glia co-cultures but not in neuron-enriched cultures. It is well known that neuroinflammation is an inevitable pathological process implicated in all types of neurological disorders [29]. The characteristic of neuroinflammation is glia activation, including microglia and astrocyte activation. Upon activation by brain damage, inflammation, and pathogens, glia cells present changes in their morphology and molecular repertoire. Actually, astrocyte became hypertrophied with an increased expression of GFAP [30], while microglia came to be hypertrophied and had the increased Iba-1 expression [31]. Following these morphological changes, microglia and astrocyte are referred to be reactive or activated. Most importantly, astrocytes and microglia release a battery of inflammatory cytokines and neurotoxic factors. The cumulation of these factors caused injury to the surrounding neuronal, particularly DA neuronal damage [32]. However, the continuous death of DA neurons, in turn lead to secondary activation of glia cells and the activated glia cells further secreted pro-inflammatory factors and thus caused DA neuronal loss [33]. Collectively, a vicious cycle to induce the prolonged neuroinflammation and the progressive reduction of DA neurons was created [34]. Therefore, attenuating activation of glia cells-elicited neuroinflammation might hold a promising strategy for the treatment of neuroinflammation-related disorders. In this study, ICA suppressed 6-OHDA-induced Iba-1 and GFAP activation and then reduced pro-inflammatory factors secretion. On the other hand, another result should be mentioned. Besides the involvement in the neuroinflammation, astroglia was the most abundant glial cells type in brain, participated in growth, nutrition, and repair of the nervous system [35]. Most importantly, astroglia are the major source of various neurotrophic factors, such as GDNF and BDNF. These neurotrophic factors have been confirmed to repair neurons and exert neuroprotection [36]. However, this study found that ICA inhibited 6-OHDA-induced GFAP activation but had no effect on BDNF and GDNF production, 


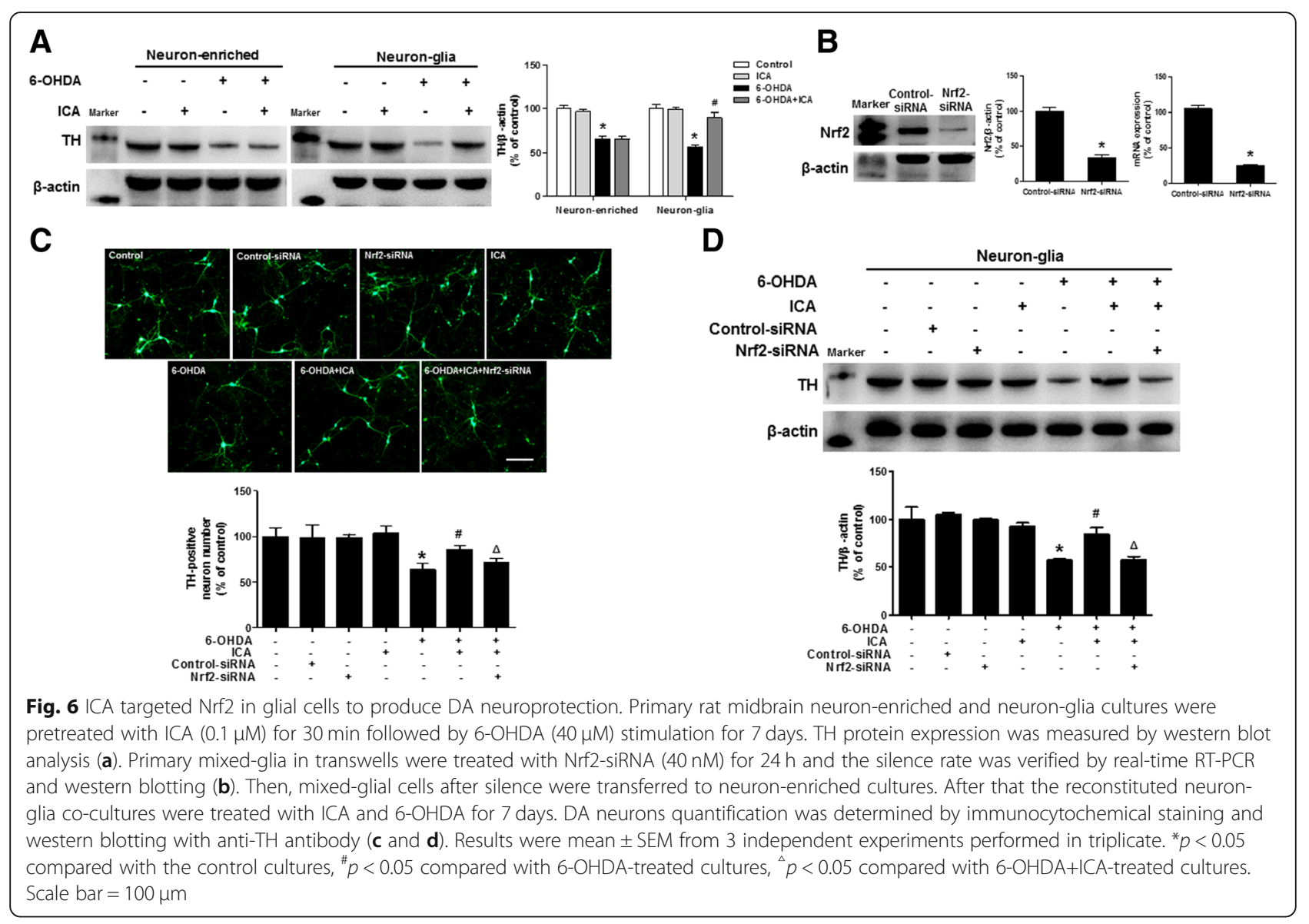

suggesting that astroglia-derived neurotrophic effects were not associated with ICA-mediated neuroprotection in this model.

Furthermore, new findings have contacted activation of Nrf2 signaling with anti-inflammatory activities. Activation of Nrf2 signaling could reduce the overproduction of pro-inflammatory factors to ameliorate the progression of rheumatoid arthritis [37]. In Nrf2 knockout mice, the exacerbation of DA neurodegeneration in $\mathrm{SN}$ and increased content of $\alpha$-synuclein were indicated, which coordinated to aggravate DA neurons loss and neuroinflammation in PD early-stage [38]. Together, Nrf2 activation not only produced anti-oxidative effects, but also regulated redox homoeostasis and attenuated neuroinflammatory responses and thus conferred neuroprotection. Here, this study indicated that ICA inhibited pro-inflammatory factors release during 6-OHDA-induced DA neuronal loss, whereas these inhibitory effects were not demonstrated in Nrf2 knockout mice, implying that the activation of $\mathrm{Nrf} 2$ signaling was involved in ICA-mediated anti-neuroinflammatory actions.

Based on the mechanistic studies mentioned above, we suggest that ICA suppressed neuroinflammatory responses and further produced DA neuroprotection through the activation of $\mathrm{Nrf} 2$ signaling.

\section{Conclusion}

This study demonstrated that ICA attenuated glia cells-mediated neuroinflammation and evoked DA neuroprotection via an Nrf2-dependent manner. These findings might provide new clues for further investigating the mechanisms underlying Nrf2-mediated neuroprotection and other Nrf2-mediated processes.

\section{Additional file}

Additional file 1: Figure S1. ICA protected 6-OHDA-induced DA neuronal damage in WT mice and Nrf2 KO mice. The administration of WT mice and $\mathrm{Nrf2} \mathrm{KO}$ mice as described in Figs. 1 and 3. We combined WT and Nrf2 KO mice to detect the protein expression of $\mathrm{TH}$ by western blot assay. Results were mean \pm SEM from 5 to 6 mice. ${ }^{*} p<0.05$ compared with control group in WT mice, ${ }^{\#} p<0.05$ compared with 6-OHDA group in WT mice, ${ }^{{ }^{*}} p<0.05$ compared with control group in Nrf2 KO mice, ${ }^{\Delta} p<0.05$ compared with 6OHDA group in WT mice. (TIF $3082 \mathrm{~kb}$ )

\section{Abbreviations}

6-OHDA: 6-hydroxydopamine; AD: Alzheimer's disease; ALS: Amyotrophic lateral sclerosis; BDNF: Brain-derived neurotrophic factor; DA: Dopamine; DMF: Dimethyl fumarate; GDNF: Glia cells line-derived neurotrophic factor; GFAP: Glia fibrillary acidic protein; HD: Huntington's disease; HO-1: Heme oxygenase-1; Iba-1: Ionized calcium-binding adapter molecule-1; ICA: Icariin; IL-1 $\beta$ : Interleukin-1 $\beta$; iNOS: Inducible nitric oxide synthase; Keap1: Kelch-like ECH-associated protein 1; LPS: Lipopolysaccharide; NO: Nitric oxide; NQO1: NADPH quinone oxidoreductase 1; Nrf2 KO: Nrf2 knockout; 
Nrf2: Nuclear factor erythroid-2 related factor 2; PCNA: Proliferating cell nuclear antigen; PD: Parkinson's disease; RNS: Reactive nitrogen species; ROS: Reactive oxygen species; SN: Substantia nigra; TH: Tyrosine hydroxylase; TNF-a: Tumor necrosis factor-a; WT: Wild type

\section{Acknowledgements}

We are grateful to the technical support provided by Shanghai Baiqu Biomedical Technology Co., Ltd.

\section{Funding}

This study was supported by the National Natural Science Foundation of China (No. 81760658), the foundation for High-level Innovative Talents of Guizhou Province (No. 20164027), Shijingshan's Tutor Studio of Pharmacology (No. GZS-201607), the Innovation Research Group project of Education Department of Guizhou Province (No. 2016038), the foundation for Excellent Young Talents of Zunyi Medical University (No.201603) and Master Start Foundation of Zunyi Medical University (no. F-898).

\section{Availability of data and materials}

The datasets used and/or analyzed during the current study are available from the corresponding author on reasonable request.

\section{Authors' contributions}

FZ conceived and designed the experiments. All the authors participated in the experiment performance and data analysis. FZ, BZ, and GQW wrote, revised, and checked the article. All authors read, revised, and approved the final manuscript.

\section{Ethics approval and consent to participate}

All experimental procedures were carried out in accordance with Chinese Guidelines of Animal Care and Welfare and this study received an approval from the Animal Care and Use Committee of Zunyi Medical University (Zunyi, China).

\section{Consent for publication}

Not applicable.

\section{Competing interests}

The authors declare that they have no competing interests.

\section{Publisher's Note}

Springer Nature remains neutral with regard to jurisdictional claims in published maps and institutional affiliations.

Received: 11 February 2019 Accepted: 1 April 2019

Published online: 22 April 2019

\section{References}

1. Panicker $N$, Saminathan $H_{\text {, Jin }} H$, Neal M, Harischandra DS, Gordon $R$, et al. Fyn kinase regulates microglial Neuroinflammatory responses in cell culture and animal models of Parkinson's disease. J Neurosci. 2015;35:10058-77.

2. Maiti P, Manna J, Dunbar GL. Current understanding of the molecular mechanisms in Parkinson's disease: targets for potential treatments. Transl Neurodegener. 2017;6:28

3. Tansey MG, McCoy MK, Frank-Cannon TC. Neuroinflammatory mechanisms in Parkinson's disease: potential environmental triggers, pathways, and targets for early therapeutic intervention. Exp Neurol. 2007;208:1-25.

4. AIDakheel A, Kalia LV, Lang AE. Pathogenesis-targeted, disease-modifying therapies in Parkinson disease. Neurotherapeutics. 2014;11:6-23.

5. Yan J, Li J, Zhang L, Sun Y, Jiang J, Huang Y, et al. Nrf2 protects against acute lung injury and inflammation by modulating TLR4 and Akt signaling. Free Radic Biol Med. 2018:121:78-85.

6. Ruiz S, Pergola PE, Zager RA, Vaziri ND. Targeting the transcription factor Nrf2 to ameliorate oxidative stress and inflammation in chronic kidney disease. Kidney Int. 2013;83:1029-41.

7. Zhao Y, Song W, Wang Z, Wang Z, Jin X, Xu J, et al. Resveratrol attenuates testicular apoptosis in type 1 diabetic mice: role of Akt-mediated Nrf2 activation and p62-dependent Keap1 degradation. Redox Biol. 2017;14:609-17.

8. Dheen ST, Jun Y, Yan Z, Tay SS, Ling EA. Retinoic acid inhibits expression of TNF-alpha and iNOS in activated rat microglia. Glia. 2005;50:21-31.
9. Lastres BI, Garcia-Yague AJ, Scannevin RH, Casarejos MJ, Kügler S, Rábano A, et al. Repurposing the NRF2 activator dimethyl fumarate as therapy against synucleinopathy in Parkinson's disease. Antioxid Redox Signal. 2016;25:61-77.

10. Vargas MR, Mariana P, Patricia C, Beckman JS, Luis B. Increased glutathione biosynthesis by Nrf2 activation in astrocytes prevents p75NTR-dependent motor neuron apoptosis. J Neurochem. 2010;97:687-96.

11. Neymotin A, Calingasan NY, Wille E, Naseri N, Petri S, Damiano M, et al. Neuroprotective effect of Nrf2/ARE activators, CDDO ethylamide and CDDO trifluoroethylamide, in a mouse model of amyotrophic lateral sclerosis. Free Radic Biol Med. 2011;51:88-96.

12. Li C, Li Q, Mei Q, Lu T. Pharmacological effects and pharmacokinetic properties of icariin, the major bioactive component in Herba Epimedii. Life Sci. 2015:126:57-68.

13. Song YH, Cai H, Zhao ZM, Chang WJ, Gu N, Cao SP, et al. Icariin attenuated oxidative stress induced-cardiac apoptosis by mitochondria protection and ERK activation. Biomed Pharmacother. 2016;83:1089-94.

14. Liu B, Xu C, Wu X, Liu F, Du Y, Sun J, et al. Icariin exerts an antidepressant effect in an unpredictable chronic mild stress model of depression in rats and is associated with the regulation of hippocampal neuroinflammation. Neuroscience. 2015;294:193-205.

15. Wang GQ, Li DD, Huang C, Lu DS, Zhang C, Zhou SY, et al. Icariin reduces dopaminergic neuronal loss and microglia-mediated inflammation in vivo and in vitro. Front Mol Neurosci. 2017;10:441.

16. Latchoumycandane C, Anantharam V, Jin H, Kanthasamy A, Kanthasamy A. Dopaminergic neurotoxicant 6-OHDA induces oxidative damage through proteolytic activation of PKCdelta in cell culture and animal models of Parkinson's disease. Toxicol Appl Pharmacol. 2011;256:314-23.

17. Wang T, Wang L, Li C, Han B, Wang Z, Li J, et al. Hydroxysafflor yellow a improves motor dysfunction in the rotenone-induced mice model of Parkinson's disease. Neurochem Res. 2017:42:1325-32.

18. Su RJ, Zhen JL, Wang W, Zhang JL, Zheng Y, Wang XM. Time-course behavioral features are correlated with Parkinson's diseaseassociated pathology in a 6-hydroxydopamine hemiparkinsonian rat model. Mol Med Rep. 2018;17:3356-63.

19. Zhang B, Wei YZ, Wang GQ, Li DD, Shi JS, Zhang F. Targeting MAPK pathways by Naringenin modulates microglia M1/M2 polarization in lipopolysaccharide-stimulated cultures. Front Cell Neurosci. 2019;12:531.

20. Zhang F, Qian L, Flood PM, Shi JS, Hong JS, Gao HM. Inhibition of IkappaB kinase-beta protects dopamine neurons against lipopolysaccharide-induced neurotoxicity. J Pharmacol Exp Ther. 2010;333:822-33.

21. Liu B, Du L, Hong JS. Naloxone protects rat dopaminergic neurons against inflammatory damage through inhibition of microglia activation and superoxide generation. J Pharmacol Exp Ther. 2000;293:607-17.

22. Sugimoto N, Toma T, Shimizu M, Kuroda M, Wada T, Yachie A. Shiga toxin-2 enhances heat-shock-induced apoptotic cell death in cultured and primary glial cells. Cell Bio Toxicol. 2014;30:289-99.

23. Méndezarmenta M, Navaruíz C, Juárezrebollar D, Rodríguezmartínez E, Gómez PY. Oxidative stress associated with neuronal apoptosis in experimental models of epilepsy. Oxidative Med Cell Longev. 2014;2014:293689.

24. Liu Z, Zhou T, Ziegler AC, Dimitrion P, Zuo L. Oxidative stress in neurodegenerative diseases: from molecular mechanisms to clinical applications. Oxidative Med Cell Longev. 2017;2017:1-11.

25. Pajares M, Jiménez-Moreno N, García-Yagüe ÁJ, Escoll M, Ceballos MLD, Leuven FV, et al. Transcription factor NFE2L2/NRF2 is a regulator of macroautophagy genes. Autophagy. 2016;12:1902-16.

26. Rojo Al, Pajares M, Rada P, Nunez A, Nevado-Holgado AJ, Killik R, et al. NRF2 deficiency replicates transcriptomic changes in Alzheimer's patients and worsens APP and TAU pathology. Redox Biol. 2017;13:444-51.

27. Shu W, Weiwei Z, Xiaolin L, Peng X, Songhui J, Daru L, et al. lodoacetic acid activates Nrf2-mediated antioxidant response in vitro and in vivo. Environ Sci Technol. 2014;48:13478-88.

28. Huanying S, Xu J, Xinbing W, Perez RG, Manru R, Xiumei Z, et al. S-allyl cysteine activates the Nrf2-dependent antioxidant response and protects neurons against ischemic injury in vitro and in vivo. J Neurochem. 2015;133:298-308.

29. Mietto BS, Mostacada K, Martinez AM. Neurotrauma and inflammation: CNS and PNS responses. Mediat Inflamm. 2015;2015:1-14.

30. Ducourneau VRR, Tiphaine D, Sabira HD, Miraucourt LSS, Aurélie A, Lucie B, et al. Cancer pain is not necessarily correlated with spinal overexpression of reactive glia markers. Pain. 2014;155:275-91.

31. Ji RR, Suter MR. p38 MAPK, microglial signaling, and neuropathic pain. Mo Pain. 2007;3:33. 
32. Tentillier N, Etzerodt A, Olesen MN, Rizalar FS, Jacobsen J, Bender D, et al. Anti-inflammatory modulation of microglia via CD163-targeted glucocorticoids protects dopaminergic neurons in the 6-OHDA Parkinson's disease model. J Neurosci. 2016:36:9375-90.

33. Block M, Zecca L, Hong J. Microglia-mediated neurotoxicity: uncovering the molecular mechanisms. Nat Rev Neurosci. 2007;8:57-69.

34. Gao HM, Hong JS. Why neurodegenerative diseases are progressive: uncontrolled inflammation drives disease progression. Trends Immunol. 2008:29:357-65.

35. Becerracalixto A, Cardonagómez GP. The role of astrocytes in neuroprotection after brain stroke: potential in cell therapy. Front Molecul Neurosci. 2017;10:88.

36. Karki P, Smith K, Johnson J Jr, Lee E. Astrocyte-derived growth factors and estrogen neuroprotection: role of transforming growth factor-a in estrogeninduced upregulation of glutamate transporters in astrocytes. Mol Cell Endocrinol. 2014;389:58-64

37. O'Connell MA, Hayes JD. The Keap1/Nrf2 pathway in health and disease: from the bench to the clinic. Biochem Soc Trans. 2015;43:687-9.

38. Isabel LB, Ayse U, Innamorato NG, Gurdal S, Alberto R, Deniz K, et al. aSynuclein expression and Nrf2 deficiency cooperate to aggravate protein aggregation, neuronal death and inflammation in early-stage Parkinson's disease. Hum Mol Genet. 2012;21:3173-92.

Ready to submit your research? Choose BMC and benefit from:

- fast, convenient online submission

- thorough peer review by experienced researchers in your field

- rapid publication on acceptance

- support for research data, including large and complex data types

- gold Open Access which fosters wider collaboration and increased citations

- maximum visibility for your research: over $100 \mathrm{M}$ website views per year

At BMC, research is always in progress.

Learn more biomedcentral.com/submissions 